\title{
Sensibilidade de mudas pré-brotadas e minirrebolos de cana-de-açúcar a herbicidas pré-emergentes
}

\author{
Paulo Vinicius da SILVA ${ }^{1 *}$, Henrique Rodrigues Milagres VIANA², Marcelo Rafael MALARDO², \\ Roque de Carvalho DIAS ${ }^{3}$, Estela Maris INÁCIO ${ }^{4}$, Nagila Moraes RIBEIRO ${ }^{5}$, \\ Patrícia Andrea MONQUERO 6 , Pedro Jacob CHRISTOFFOLETI ${ }^{2}$
}

\author{
${ }^{1}$ Universidade Federal de Grande Dourados, Dourados, MS, Brasil. \\ ${ }^{2}$ Escola Superior de Agricultura Luiz de Queiroz, Universidade de São Paulo, Piracicaba, SP, Brasil. \\ (Orcid: 0000-0002-1044-5594; 0000-0002-1076-785X; 0000-0002-2741-5615)
}

${ }^{3}$ Faculdade de Ciências Agronômicas, Universidade Estadual Paulista, Botucatu, SP, Brasil. (Orcid: 0000-0001-5433-5373)

${ }^{6}$ Setor de Ciências Agrarias e de Tecnologia, Universidade Estadual de Ponta Grossa, Ponta Grossa, PR, Brasil. (Orcid: 0000-0003-3262-2819)

${ }^{5}$ Faculdade de Ciências Agronômicas e Veterinárias, Universidade Estadual Paulista, Jaboticabal, SP, Brasil. (Orcid: 0000-0001-9258-7969)

${ }^{6}$ Centro de Ciências Agrárias, Universidade Federal de São Carlos, Araras, SP, Brasil. (Orcid: 0000-0002-9123-1861)

*E-mail: paulovsilva@ufgd.edu.br (Orcid:0000-0003-4647-5602)

Recebido em 13/04/2020; Aceito em 10/06/2020; Publicado em 31/07/2020.

\begin{abstract}
RESUMO: A cana-de-açúcar, cultivada através de minirrebolos e mudas pré-brotadas, podem apresentar maior sensibilidade a herbicidas em comparação ao convencional. Objetivou-se avaliar a seletividade inicial de tratamentos herbicidas aplicados em pré-emergência em variedades de cana-de-açúcar cultivadas nos sistemas de mudas pré-brotadas e de minirrebolos. Em casa de vegetação foram realizados dois experimentos, um com MPBs (RB966928 e IAC 5000) e outro com minirrebolos (RB92579 e RB966928). O delineamento foi inteiramente casualizado, com quatro repetições em esquema fatorial $8 \times 5$, sendo oito tratamentos (amicarbazone (980,0 $\left.\mathrm{g} \mathrm{ha}^{-1}\right)$; metribuzin $\left(921,6 \mathrm{~g} \mathrm{ha}^{-1}\right)$; indaziflam $\left(50,0 \mathrm{~g} \mathrm{ha}^{-1}\right)$; isoxaflutole $\left(78,8 \mathrm{~g} \mathrm{ha}^{-1}\right)$; amicarbazone + indaziflam $\left(703,5+37,5 \mathrm{~g} \mathrm{ha}^{-1}\right)$; metribuzin + indaziflam $\left(460,8+37,5 \mathrm{~g} \mathrm{ha}^{-1}\right)$, isoxaflutole + indaziflam $\left(56,3+37,5 \mathrm{~g} \mathrm{ha}^{-1}\right)$ e testemunha (sem herbicidas) e cinco épocas de avaliação (7, 14, 2128 e 35 dias após aplicação dos tratamentos e emergência, para MPBs e minirrebolos, respectivamente). Em MPBs, os herbicidas amicarbazone e metribuzin, resultaram em $0,00 \%$ de fitotoxicidade nas duas variedades. Nos minirrebolos, o isoxaflutole isolado e associado com indaziflam resultaram em alta fitotoxicidade independentemente da variedade. Logo o sistema de plantio e as variedades, influenciam na seletividade de herbicidas na cultura da cana-de-açúcar.
\end{abstract}

Palavras-chave: cana-planta; fitotoxicidade; aplicação; sistemas de plantio.

\section{Sensitivity of pre-budded seedlings and sugar cane multibud setts to pre- emergent herbicides}

\begin{abstract}
The sugarcane, grown through pre-budded seedlings and multibud setts, may have more sensitive to herbicides compared to conventional. The objective of this study was to evaluate the initial selectivity of pre-emergent herbicide treatment in sugarcane varieties cultivated in the pre-budded seedlings and multibud setts systems. Two experiments were carried out in the greenhouse, one with MPBs (RB966928 and IAC 5000) and the other with multibud setts (RB92579 and RB966928). The design was completely randomized, with four replications in an $8 \times 5$ factorial scheme, with eight treatments: amicarbazone $\left(1400 \mathrm{~g} \mathrm{ha}^{-1}\right)$; metribuzin (1920 $\left.\mathrm{g} \mathrm{ha}^{-1}\right)$; indaziflam (100 $\left.\mathrm{g} \mathrm{ha}^{-1}\right)$; isoxaflutole (105 $\left.\mathrm{g} \mathrm{ha}^{-1}\right)$; amicarbazone + indaziflam $\left(1005+75 \mathrm{~g} \mathrm{ha}^{-1}\right)$; metribuzin + indaziflam $\left(960+75 \mathrm{~g} \mathrm{ha}^{-1}\right)$, isoxaflutole + indaziflam $\left(75+75 \mathrm{~g} \mathrm{ha}^{-1}\right)$ and control (without herbicides) and five evaluation periods (7, 14, 21, 28 and 35 days after application of the DAT and emergency treatments, for MPBs and multibud setts, respectively). In MPBs, the herbicides amicarbazone and metribuzin, resulted in $0.00 \%$ phytotoxicity in both varieties. In multibud setts, isoxaflutole alone and associated with indaziflam resulted in high phytotoxicity regardless of variety. Soon the planting system and the varieties, influence the selectivity of herbicides in the culture of sugarcane.
\end{abstract}

Keywords: sugarcane-plant; phytotoxicity; application; planting systems.

\section{INTRODUÇÃO}

No sistema convencional de cultivo de cana-de-açúcar são utilizados cerca de 18 a 20 toneladas por hectare de colmos, os quais possuem entre $30 \mathrm{a} 40 \mathrm{~cm}$ de comprimento e são posicionados entre 30 e $40 \mathrm{~cm}$ de profundidade do solo (LANDELL et al., 2013). Esse sistema de cultivo apresenta como desvantagem, uma grande massa de material vegetal a ser utilizado no plantio, dificultando o transporte, movimentação e armazenagem, desse material, favorecendo, assim, a rápida deterioração e, consequentemente, reduzindo a viabilidade das gemas plantadas (FRAGA JÚNIOR, 2015). 
Diante desse cenário, novos sistemas de plantio de canade-açúcar surgem como alternativa para redução da massa vegetal necessária no plantio convencional, como por exemplo, o sistema de mudas pré-brotadas (MPBs) e o sistema de minirrebolos de cana-de-açúcar (MAY; RAMOS, 2019). O sistema MPB reduz a quantidade de mudas utilizadas no plantio de 10 para $2 \mathrm{t} \mathrm{ha}^{-1}$ (MAWLA et al., 2014), proporciona melhor controle de vigor e sanidade das mudas, possui maior taxa de multiplicação e uniformidade no plantio e a sua distribuição no campo permite a utilização de irrigação localizada (LANDELL et al., 2013).

No sistema de minirrebolos de cana-de-açúcar, são utilizados colmos de até $4 \mathrm{~cm}$ de comprimento contendo uma única gema (ARAUJO, 2015). Esta tecnologia de plantio simplifica e agiliza o sistema de plantio, por ser um processo totalmente mecanizado que utiliza equipamentos leves (BERTOLINO; ALVES, 2014), sendo viável mesmo sob o cultivo mínimo (MARTINHO et al., 2010). O sistema de minirrebolos reduz a quantidade de colmos utilizada no plantio de 18 para $1,5 \mathrm{t} \mathrm{ha}^{-1}$ e são plantados a $30 \mathrm{~cm}$ de profundidade recobertos com 5-8 $\mathrm{cm}$ de solo (MARTINHO et al., 2010).

Estes dois sistemas de plantio de cana-de-açúcar, apresentam diferenças em relação a sensibilidade a herbicidas, quando comparados com o sistema convencional. No sistema convencional não ocorre o contato do sistema radicular com a zona tratada de herbicidas, reduzindo os efeitos fitotóxicos desses produtos, quando aplicados nas condições corretas.

Segundo Dias et al. (2017), a localização espacial do herbicida em relação a planta é responsável pela seletividade do produto, conhecida por seletividade de posicionamento. Já nos sistemas de MPBs e minirrebolos, o sistema radicular da cana-de-açúcar é desenvolvido próximo a superfície do solo o que pode gerar problemas relacionados a seletividade de herbicidas. Além desses fatores, a seletividade pode ser diferente de acordo com a tolerância de cada variedade de cana-de-açúcar a determinados produtos herbicidas (GIRALDELI et al., 2018).
Portanto, o objetivo deste trabalho foi avaliar a seletividade inicial de tratamentos herbicidas aplicados em pré-emergência em variedades de cana-de-açúcar cultivadas nos sistemas de mudas pré-brotadas e de minirrebolos.

\section{MATERIAL E MÉTODOS}

Foram realizados dois experimentos para cada sistema de plantio. O primeiro com mudas pré-brotadas (MPBs) das variedades RB966928 e IAC 5000, e o segundo com minirrebolos das variedades RB92579 e RB966928. Ambos foram conduzidos em casa-de-vegetação seguindo $\mathrm{O}$ delineamento experimental inteiramente casualizado, com quatro repetições e esquema fatorial $8 \times 5$, sendo oito tratamentos de herbicidas (amicarbazone (980,0 $\mathrm{g} \mathrm{ha}^{-1}$ ); metribuzin $\left(921,6 \mathrm{~g} \mathrm{ha}^{-1}\right)$; indaziflam $\left(50,0 \mathrm{~g} \mathrm{ha}^{-1}\right)$; isoxaflutole $\left(78,8 \mathrm{~g} \mathrm{ha}^{-1}\right)$; amicarbazone + indaziflam $(703,5+37,5 \mathrm{~g}$ ha$\left.{ }^{1}\right)$; metribuzin + indaziflam $\left(460,8+37,5 \mathrm{~g} \mathrm{ha}^{-1}\right)$, isoxaflutole + indaziflam $\left(56,3+37,5 \mathrm{~g} \mathrm{ha}^{-1}\right)$, além da testemunha sem aplicação de herbicidas e cinco épocas de avaliação $(7,14,21$ 28 e 35 dias após aplicação dos tratamentos (DAT) para as MPBs e dias após a emergência (DAE) para os minirrebolos). Se ressalta que as unidades experimentais relativas a testemunha foram submetidas as mesmas condições de desenvolvimento dos demais tratamentos.

Os experimentos foram realizados no período de 07 de julho até 20 de outubro de 2016. As unidades experimentais foram constituídas por vasos plásticos com capacidade volumétrica de $12 \mathrm{~L}$, preenchidos com solo classificado como Latossolo Vermelho Distroférrico (EMBRAPA, 2013) oriundo da camada arável $(0,00-0,20 \mathrm{~m})$, previamente peneirado, coletado de uma área sem histórico de utilização de herbicidas. A análise do solo está expressa na Tabela 1, a qual serviu como base para adubação, a qual foi pautada nas recomendações do boletim 100, visando proporcionar as melhores condições de desenvolvimento para cana-de-açúcar (RAIJ et al., 1996).

Tabela 1. Características físico-químicos do Latossolo Vermelho Distroférrico.

Table 1. Physico-chemical characteristics of the dystrophic red oxisol.

\begin{tabular}{|c|c|c|c|c|c|c|c|c|c|c|c|c|}
\hline $\mathrm{pH}$ & $\mathrm{Al}^{3+}$ & $\mathrm{H}^{+}+\mathrm{Al}^{3+}$ & $\mathrm{Ca}^{2+}+\mathrm{Mg}^{2+}$ & $\mathrm{Ca}^{2+}$ & $\mathrm{K}^{+}$ & M.O. & $\mathrm{P}$ & CTC $(\mathrm{pH}=7)$ & V & Areia & Silte & Argila \\
\hline$\left(\mathrm{CaCl}_{2}\right)$ & & & $\left(\mathrm{mmol}_{c} \mathrm{dm}^{-3}\right)$ & & & $\left(\mathrm{g} \mathrm{dm}^{-3}\right)$ & $\left(\mathrm{mg} \mathrm{dm}^{-3}\right)$ & $\left(\mathrm{mmol}_{c} \mathrm{dm}^{-3}\right)$ & \multicolumn{4}{|c|}{$(\%)$} \\
\hline 5,3 & $<1,0$ & 25,0 & 13,0 & 26,0 & 2,8 & 17,0 & 10,0 & 68,0 & 63,0 & 41,0 & 5,0 & 54,0 \\
\hline
\end{tabular}

Para o plantio das MPBs, os vasos foram preenchidos com solo e, após aplicação individual dos tratamentos de herbicidas (na condição de pré-plantio), foram abertos sulcos de cultivo nos vasos onde as mudas foram posicionadas.

Já para o plantio dos minirrebolos de cana-de-açúcar, os vasos foram preenchidos com solo e os minirrebolos contendo as gemas individuais foram posicionados a $2,5 \mathrm{~cm}$ de profundidade. Após o plantio foi realizado a aplicação dos tratamentos de herbicidas em solo úmido.

As aplicações foram realizadas utilizando pulverizador costal pressurizado por $\mathrm{CO}_{2}$, com pressão constante de 2,5 $\mathrm{kgf} \mathrm{cm}{ }^{-2}$, com barra de aplicação provida de quatro bicos com pontas de pulverização do tipo leque TTI 110.03, com uma vazão de $150 \mathrm{~L} \mathrm{ha}^{-1}$ de calda. As condições climáticas no momento da aplicação foram: umidade relativa do ar de
$65,0 \%$, temperatura de $26,6^{\circ} \mathrm{C}$ e a velocidade do vento de 2,3 $\mathrm{km} \mathrm{h}^{-1}$.

Após a aplicação dos tratamentos as unidades experimentais foram mantidas em casa-de-vegetação com sistema de irrigação por aspersão, irrigadas diariamente com $10 \mathrm{~mm} \mathrm{dia}{ }^{-1}$. As avaliações visuais de fitotoxicidade foram realizadas aos 7, 14, 21, 28 e 35 dias após aplicação dos tratamentos (DAT) para as mudas pré-brotadas (MPBs) e 7 , 14, 21, 28 e 35 dias após a emergência da cana-de-açúcar (DAE) para os minirrebolos. A escala de notas utilizadas foi a proposta pela European Weed Research Council, onde 0\% está relacionado com a ausência de dano e 100\% significa destruição total das plantas (morte da planta) (EWRC, 1964).

Aos 35 DAT (para MPBs) e 35 DAE (para minirrebolos), foram realizadas as seguintes avaliações biométricas: (1) Altura das plantas de cana-de-açúcar; (2) Diâmetro de 
colmos; (3) Nível de clorofila total $(a+b)$ através de um medidor de clorofila SPAD-502 Plus; (4) Análise da área foliar utilizando o aparelho Licor-3100; (5) Massa seca da parte aérea e (6) Massa seca do sistema radicular. As secções das plantas (parte aérea e sistema radicular) foram acondicionados em sacos de papel de forma separada e levados para estufa de circulação forçada de ar com temperatura de $60^{\circ} \mathrm{C}+/-2^{\circ} \mathrm{C}$ até atingirem peso constante.

Todos os dados foram submetidos à análise de variância pelo teste $\mathrm{F}$, e as médias foram comparadas por meio do teste de Tukey, utilizando-se o programa estatístico computacional AgroEstat (BARBOSA; MALDONADO JÚNIOR, 2015).

\section{RESULTADOS}

Os resultados de porcentagem de fitotoxicidade obtidos para as MPBs da variedade RB966928 estão expressos na Tabela 2. Observa-se que aos 7 DAT, apenas os herbicidas amicarbazone e metribuzin causaram injúrias nas MPBs, porém não significativo em relação aos demais tratamentos. Aos 14 DAT, as MPBs submetidas aos tratamentos com amicarbazone e metribuzin se recuperaram da fitotoxicidade causada aos 7 DAT, enquanto que os demais tratamentos causaram efeitos fitotóxicos nas mudas pré-brotadas de canade-açúcar. A partir dos 21 DAT apenas os tratamentos com amicarbazone e metribuzin não causaram injúrias significativas nas MPBs, sendo iguais estatisticamente a testemunha sem aplicação, enquanto que nos demais tratamentos foi constatado evolução nos sintomas. Aos 35 DAT, amicarbazone e metribuzin proporcionaram $0,00 \%$ de fitotoxicidade nas MPBs, isoxaflutole, amicarbazone + indaziflam e isoxaflutole + indaziflam causaram fitotoxicidade de 70,00; 68,75 e 61,25\%, respectivamente, e os tratamentos com indaziflam e metribuzin + indaziflam causaram 93,75 e $85,00 \%$ de fitotoxicidade, respectivamente (Tabela 2).

Tabela 2. Fitotoxicidade (\%) de herbicidas pré-emergentes em MPBs de cana-de-açúcar da variedade RB966928.

Table 2. Phytotoxicity (\%) of pre-emergent herbicides in sugarcane MPBs of the RB966928 variety.

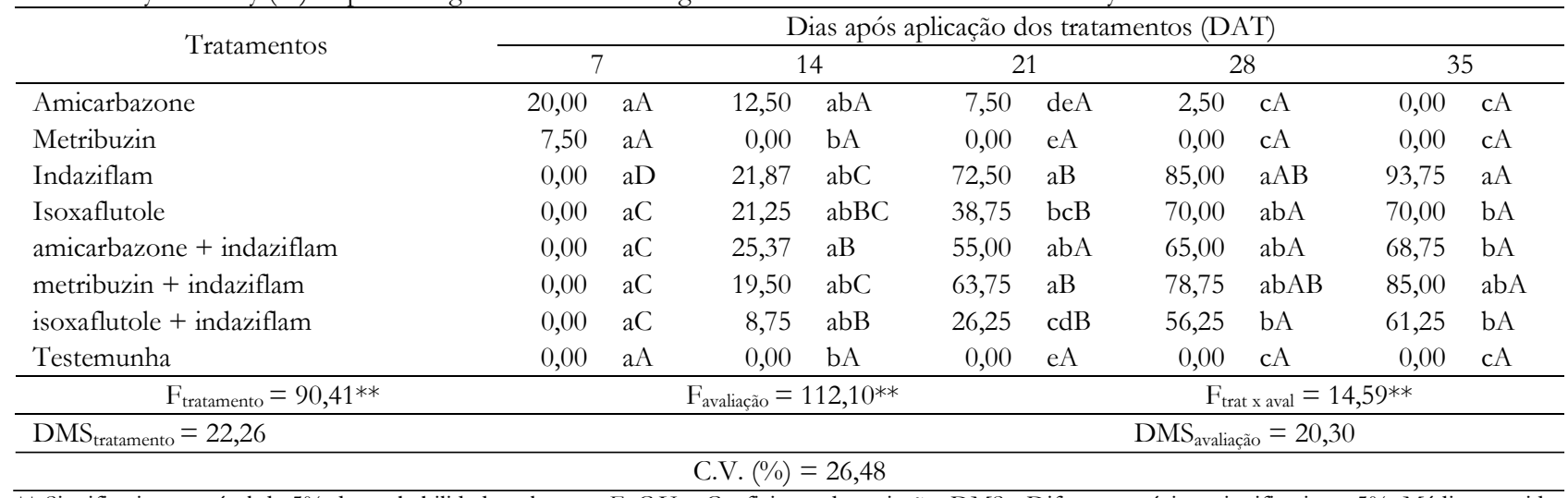

** Significativo ao nível de 5\% de probabilidade pelo teste F; C.V. - Coeficiente de variação. DMS - Diferença mínima significativa a 5\%. Médias seguidas por letras iguais, minúsculas na coluna e maiúsculas na linha, não diferem entre si pelo teste de Tukey a $5 \%$ de significância.

Com relação a fitotoxicidade em MPBs da variedade IAC 5000, os resultados estão apresentados na Tabela 3 .

Nota-se que aos 7 DAT, apenas o herbicida amicarbazone causou fitotoxicidade na cultura (20,62\%). Aos 14 DAT, a fitotoxicidade causada pelo amicarbazone reduziu para $9,00 \%$ e a pelo metribuzin continuou em $0,00 \%$, enquanto que os demais tratamentos causaram fitotoxicidade superior a $20,00 \%$, com exceção do isoxaflutole que causou apenas $2,50 \%$ de fitotoxicidade. Assim como foi observado para a variedade RB966928, na variedade IAC 5000, a partir dos 21 DAT, a fitotoxicidade causada pelo herbicida amicarbazone continuou diminuindo e o tratamento com metribuzin permaneceu em $0,00 \%$, já para os demais tratamentos notou-se evolução dos sintomas fitotóxicos. Aos 35 DAT, os tratamentos contendo os herbicidas isoxaflutole isolado e em associação com indaziflam causaram fitotoxicidade de 66,25 e 68,75\%, respectivamente, enquanto que os tratamentos com indaziflam isolado e em associação com amicarbazone e metribuzin proporcionaram efeitos fitotóxicos de 86,$25 ; 81,25$ e $80,00 \%$, respectivamente (Tabela 3).

Tabela 3. Fitotoxicidade (\%) de herbicidas pré-emergentes em MPBs de cana-de-açúcar da variedade IAC 5000.

Table 3. Phytotoxicity (\%) of pre-emergent herbicides in sugarcane MPBs of the IAC5000 variety.

\begin{tabular}{|c|c|c|c|c|c|c|c|c|c|c|}
\hline \multirow{2}{*}{ Tratamentos } & \multicolumn{10}{|c|}{ Dias após aplicação dos tratamentos (DAT) } \\
\hline & \multicolumn{2}{|c|}{7} & \multicolumn{2}{|c|}{14} & \multicolumn{2}{|c|}{21} & \multicolumn{2}{|c|}{28} & \multicolumn{2}{|c|}{35} \\
\hline Amicarbazone & 20,62 & $\mathrm{aA}$ & 9,00 & $\mathrm{bB}$ & 6,40 & $\mathrm{eB}$ & 2,75 & $\mathrm{~dB}$ & 0,00 & $\mathrm{cB}$ \\
\hline Metribuzin & 0,00 & $\mathrm{bA}$ & 0,00 & bA & 0,00 & eA & 0,00 & dA & 0,00 & $\mathrm{cA}$ \\
\hline Indaziflam & 0,00 & $\mathrm{bC}$ & 32,12 & $\mathrm{aB}$ & 80,00 & $\mathrm{aA}$ & 83,75 & $\mathrm{aA}$ & 86,25 & $\mathrm{aA}$ \\
\hline Isoxaflutole & 0,00 & $\mathrm{bC}$ & 2,50 & $\mathrm{bC}$ & 23,75 & $\mathrm{~dB}$ & 66,25 & $\mathrm{bcA}$ & 66,25 & $\mathrm{bA}$ \\
\hline amicarbazone + indaziflam & 0,00 & $\mathrm{bD}$ & 29,37 & $\mathrm{aC}$ & 62,50 & bB & 75,00 & $a b A$ & 81,25 & $\mathrm{aA}$ \\
\hline metribuzin + indaziflam & 0,00 & bE & 27,62 & $\mathrm{aD}$ & 45,00 & $\mathrm{cC}$ & 66,25 & $\mathrm{bcB}$ & 80,00 & $\mathrm{aA}$ \\
\hline isoxaflutole + indaziflam & 0,00 & $\mathrm{bC}$ & 21,92 & $\mathrm{aB}$ & 30,00 & $\mathrm{~dB}$ & 63,75 & $\mathrm{cA}$ & 68,75 & $\mathrm{bA}$ \\
\hline Testemunha & 0,00 & $\mathrm{bA}$ & 0,00 & $\mathrm{bA}$ & 0,00 & $\mathrm{eA}$ & 0,00 & $\mathrm{dA}$ & 0,00 & $\mathrm{cA}$ \\
\hline $\mathrm{F}_{\text {tratamento }}=437,98^{* *}$ & \multicolumn{5}{|c|}{$\mathrm{F}_{\text {avaliacão }}=507,50^{* *}$} & \multicolumn{5}{|c|}{$\mathrm{F}_{\text {trat } \mathrm{x} \text { aval }}=65,04^{* *}$} \\
\hline $\mathrm{DMS}_{\text {tratamento }}=10,56$ & & & & & & & $\mathrm{MS}_{\text {avalia }}$ & à $=9$ & & \\
\hline \multicolumn{11}{|c|}{ C.V. $(\%)=16,39$} \\
\hline
\end{tabular}

** Significativo ao nível de 5\% de probabilidade pelo teste F; C.V. - Coeficiente de variação. DMS - Diferença mínima significativa a 5\%. Médias seguidas por letras iguais, minúsculas na coluna e maiúsculas na linha, não diferem entre si pelo teste de Tukey a $5 \%$ de significância. 
As avaliações biométricas das mudas pré-brotadas de cana-de-açúcar da variedade RB966928 estão expressas na Tabela 4. Nota-se que para a avaliação de altura, apenas os tratamentos contendo amicarbazone, metribuzin e amicarbazone + indaziflam não diferiram estatisticamente da testemunha sem aplicação de herbicida, enquanto os demais tratamentos causaram redução na altura das mudas. Para o diâmetro dos colmos, apenas os tratamentos com amicarbazone e metribuzin não diferiram estatisticamente da testemunha, não havendo redução de diâmetro para as mudas pré-brotadas de cana-de-açúcar aplicadas com esses herbicidas. Com relação a avaliação de clorofila, apenas os tratamentos com indaziflam e isoxaflutole foram diferentes da testemunha, havendo redução nos teores de clorofila para estes tratamentos. Para análise de área foliar, os tratamentos com amicarbazone, metribuzin e amicarbazone + indaziflam não diferiram estatisticamente da testemunha, enquanto os demais tratamentos causaram redução significativa da área foliar. Com relação a matéria seca da parte aérea, os herbicidas amicarbazone e metribuzin foram os únicos tratamentos que não causaram redução da massa seca da parte aérea, sendo iguais estatisticamente a testemunha. Por último, a análise de matéria seca do sistema radicular mostrou que houve diferença estatística apenas entre o tratamento com metribuzin, em relação aos tratamentos com indaziflam, isoxaflutole e metribuzin + indaziflam (Tabela 4).

Tabela 4. Avaliações biométricas aos 35 DAT, de MPBs da variedade RB966928, após aplicação de herbicidas em pré-emergência. Table 4. Biometric evaluations at 35 DAT, of MPBs of the RB966928 variety, after application of herbicides in pre-emergence.

\begin{tabular}{|c|c|c|c|c|c|c|c|c|c|c|c|c|}
\hline Tratamentos & \multicolumn{2}{|c|}{$\begin{array}{c}\text { Altura } \\
(\mathrm{cm})\end{array}$} & \multicolumn{2}{|c|}{$\begin{array}{l}\text { Diâmetro } \\
(\mathrm{cm})\end{array}$} & \multicolumn{2}{|c|}{$\begin{array}{c}\text { Clorofila } \\
\left(\mathrm{mg} \mathrm{cm}^{-2}\right)\end{array}$} & \multicolumn{2}{|c|}{$\begin{array}{l}\mathrm{AF}^{1} \\
\left(\mathrm{~cm}^{2}\right)\end{array}$} & \multicolumn{2}{|c|}{$\begin{array}{c}\mathrm{MSPA}^{2} \\
(\mathrm{~g})\end{array}$} & \multicolumn{2}{|c|}{$\begin{array}{c}\mathrm{MSSR}^{3} \\
(\mathrm{~g})\end{array}$} \\
\hline amicarbazone & 77,25 & $\mathrm{a}$ & 6,75 & $a b c$ & 56,20 & a & 239,39 & $\mathrm{a}$ & 2,64 & $\bar{a}$ & 0,45 & $\mathrm{ab}$ \\
\hline metribuzin & 73,00 & $\mathrm{a}$ & 7,50 & a & 42,50 & $a b$ & 224,14 & $a b$ & 2,71 & a & 0,49 & $\mathrm{a}$ \\
\hline indaziflam & 23,00 & $\mathrm{c}$ & 2,50 & $\mathrm{e}$ & 20,72 & $\mathrm{c}$ & 10,98 & $\mathrm{~d}$ & 0,65 & $\mathrm{~b}$ & 0,10 & $\mathrm{~b}$ \\
\hline isoxaflutole & 41,25 & bc & 4,75 & $\mathrm{~d}$ & 22,87 & c & 86,64 & $\mathrm{~cd}$ & 0,89 & $\mathrm{~b}$ & 0,11 & $\mathrm{~b}$ \\
\hline amicarbazone +indaziflam & 63,50 & $a b$ & 5,75 & bcd & 28,07 & bc & 141,63 & bc & 1,07 & $\mathrm{~b}$ & 0,18 & $a b$ \\
\hline metribuzin + indaziflam & 27,75 & $\mathrm{c}$ & 5,25 & $\mathrm{~cd}$ & 42,32 & $a b$ & 17,50 & $\mathrm{~d}$ & 1,04 & $\mathrm{~b}$ & 0,12 & $\mathrm{~b}$ \\
\hline isoxaflutole + indaziflam & 30,25 & $\mathrm{c}$ & 5,25 & $\mathrm{Cd}$ & 44,95 & $a b$ & 57,55 & $\mathrm{~cd}$ & 1,38 & b & 0,20 & $a b$ \\
\hline testemunha & 77,75 & $\mathrm{a}$ & 7,25 & $\mathrm{~A}$ & 45,10 & $\mathrm{ab}$ & 220,73 & $\mathrm{ab}$ & 2,30 & $\mathrm{a}$ & 0,42 & $\mathrm{ab}$ \\
\hline $\mathrm{F}$ & \multicolumn{2}{|c|}{$16,51^{* *}$} & \multicolumn{2}{|c|}{$20,02^{* *}$} & \multicolumn{2}{|c|}{$11,15^{* *}$} & \multicolumn{2}{|c|}{$26,64 * *$} & \multicolumn{2}{|c|}{$16,03 * *$} & \multicolumn{2}{|c|}{$3,72 *$} \\
\hline DMS & \multicolumn{2}{|c|}{27,17} & \multicolumn{2}{|c|}{1,69} & \multicolumn{2}{|c|}{17,53} & \multicolumn{2}{|c|}{86,04} & & \multicolumn{2}{|c|}{0,36} \\
\hline C.V. $(\%)$ & \multicolumn{2}{|c|}{22,40} & \multicolumn{2}{|c|}{1283} & \multicolumn{2}{|c|}{1980} & \multicolumn{2}{|c|}{19,34} & & \multicolumn{2}{|c|}{25,73} \\
\hline
\end{tabular}

${ }^{1} \mathrm{AF}$ - Área foliar; ${ }^{2} \mathrm{MSPA}$ - Massa seca da parte aérea; ${ }^{3}$ MSSR - Massa seca do sistema radicular. ${ }^{* *}$ Significativo ao nível de $5 \%$ de probabilidade pelo teste F; * Significativo ao nível de 1\% de probabilidade pelo teste F; C.V. - Coeficiente de variação. . DMS - Diferença mínima significativa a 5\%. Médias seguidas por letras iguais não diferem entre si pelo teste de Tukey a 5\% de significância.

Já com relação as mudas pré-brotadas da variedade IAC 5000, as avaliações biométricas estão apresentadas na Tabela 5.

Observa-se que apenas os tratamentos com aplicação de amicarbazone e de metribuzin foram iguais estatisticamente a testemunha, para a variável altura, evidenciando que estes tratamentos não diminuíram a altura das mudas. Para a variável diâmetro, juntamente aos herbicidas amicarbazone e metribuzin, os tratamentos com o herbicida isoxaflutole e com a mistura de amicarbazone + indaziflam, também foram similares estatisticamente a testemunha não tratada. Com relação a ao teor de clorofila nas folhas das mudas, todos os tratamentos foram estatisticamente iguais a testemunha. Vale ressaltar que houve diferença estatística do tratamento com aplicação de metribuzin $\left(44,30 \mathrm{mg} \mathrm{cm}^{-2}\right)$, quando comparado com os tratamentos contendo indaziflam isolado $(19,87 \mathrm{mg}$ $\left.\mathrm{cm}^{-2}\right)$ e em associação com metribuzin $(20,92 \mathrm{mg} \mathrm{cm}-2)$. Para área foliar, observa-se que apenas os tratamentos de amicarbazone e metribuzin não diferiram estatisticamente da testemunha. O mesmo foi observado para a análise de matéria seca da parte aérea, evidenciando que estes dois tratamentos não interferiram negativamente no desenvolvimento das mudas. Já para a análise de matéria seca do sistema radicular, apenas os tratamentos com indaziflam isolado e em associação com amicarbazone e metribuzin, diferiram estatisticamente do tratamento testemunha, havendo diminuição do peso do sistema radicular para estes tratamentos (Tabela 5).

Tabela 5. Avaliações biométricas aos 35 DAT, de MPBs da variedade IAC 5000, após aplicação de herbicidas em pré-emergência. Table 5. Biometric evaluations at $35 \mathrm{DAT}$, of MPBs of the IAC5000 variety, after application of herbicides in pre-emergence.

\begin{tabular}{|c|c|c|c|c|c|c|c|c|c|c|c|c|}
\hline Tratamentos & \multicolumn{2}{|c|}{$\begin{array}{l}\text { Altura } \\
(\mathrm{cm})\end{array}$} & \multicolumn{2}{|c|}{$\begin{array}{c}\text { Diâmetro } \\
(\mathrm{cm})\end{array}$} & \multicolumn{2}{|c|}{$\begin{array}{l}\text { Clorofila } \\
\left(\mathrm{mg} \mathrm{cm}^{-2}\right)\end{array}$} & \multicolumn{2}{|c|}{$\begin{array}{l}\mathrm{AF}^{1} \\
\left(\mathrm{~cm}^{2}\right)\end{array}$} & \multicolumn{2}{|c|}{$\begin{array}{c}\text { MSPA }^{2} \\
\text { (g) }\end{array}$} & \multicolumn{2}{|c|}{$\begin{array}{c}\text { MSSR }^{3} \\
(\mathrm{~g})\end{array}$} \\
\hline amicarbazone & 75,25 & $\mathrm{a}$ & 7,00 & A & 41,47 & $a b$ & 193,08 & $\mathrm{a}$ & 2,47 & $\mathrm{a}$ & 0,60 & A \\
\hline metribuzin & 77,25 & $\mathrm{a}$ & 7,50 & A & 44,30 & $\mathrm{a}$ & 185,89 & a & 2,78 & a & 0,56 & $a b$ \\
\hline indaziflam & 21,75 & $\mathrm{c}$ & 1,00 & $\mathrm{D}$ & 19,87 & $\mathrm{~b}$ & 14,83 & $\mathrm{c}$ & 0,73 & $\mathrm{~b}$ & 0,25 & bcd \\
\hline isoxaflutole & 55,50 & $\mathrm{~b}$ & 7,50 & A & 27,40 & $a b$ & 109,58 & b & 1,41 & $\mathrm{~b}$ & 0,46 & $a b c$ \\
\hline amicarbazone +indaziflam & 52,25 & $\mathrm{~b}$ & 6,25 & $a b$ & 22,37 & $a b$ & 119,80 & $\mathrm{~b}$ & 0,90 & $\mathrm{~b}$ & 0,23 & $\mathrm{~cd}$ \\
\hline metribuzin + indaziflam & 25,75 & $\mathrm{c}$ & 4,25 & bc & 20,92 & $\mathrm{~b}$ & 25,49 & $\mathrm{c}$ & 0,94 & $\mathrm{~b}$ & 0,11 & $\mathrm{~d}$ \\
\hline isoxaflutole + indaziflam & 28,00 & $\mathrm{c}$ & 3,75 & $\mathrm{C}$ & 29,72 & $a b$ & 15,81 & c & 1,31 & $\mathrm{~b}$ & 0,49 & $\mathrm{abc}$ \\
\hline testemunha & 72,50 & $\mathrm{a}$ & 7,25 & A & 41,27 & $\mathrm{ab}$ & 183,50 & $\mathrm{a}$ & 2,51 & $\mathrm{a}$ & 0,65 & $\mathrm{a}$ \\
\hline $\mathrm{F}$ & \multicolumn{2}{|c|}{$103,41 * *$} & \multicolumn{2}{|c|}{$25,29 * *$} & \multicolumn{2}{|c|}{$4,25^{* *}$} & \multicolumn{2}{|c|}{$31,06^{* *}$} & \multicolumn{2}{|c|}{$19,11^{* *}$} & \multicolumn{2}{|c|}{$6,74 *$} \\
\hline DMS & \multicolumn{2}{|c|}{10,70} & \multicolumn{2}{|c|}{2,19} & \multicolumn{2}{|c|}{22,83} & \multicolumn{2}{|c|}{61,08} & \multicolumn{2}{|c|}{0,79} & \multicolumn{2}{|c|}{0,31} \\
\hline C.V. $(\%)$ & \multicolumn{2}{|c|}{8,95} & \multicolumn{2}{|c|}{16,81} & \multicolumn{2}{|c|}{21,53} & \multicolumn{2}{|c|}{26,46} & \multicolumn{2}{|c|}{22,56} & \multicolumn{2}{|c|}{25,02} \\
\hline
\end{tabular}

${ }^{1} \mathrm{AF}$ - Área foliar; ${ }^{2} \mathrm{MSPA}$ - Massa seca da parte aérea; ${ }^{3}$ MSSR - Massa seca do sistema radicular. ** Significativo ao nível de $5 \%$ de probabilidade pelo teste F; * Significativo ao nível de $1 \%$ de probabilidade pelo teste F; C.V. - Coeficiente de variação. . DMS - Diferença mínima significativa a $5 \%$. Médias seguidas por letras iguais não diferem entre si pelo teste de Tukey a $5 \%$ de significância. 
Para os experimentos realizados com minirrebolos de cana-de-açúcar, os resultados de fitotoxicidade obtidos para a variedade RB92579 estão expressos na Tabela 6.

Nota-se que em todas as épocas de avaliação, apenas os tratamentos com aplicação de isoxaflutole isolado e em associação com indaziflam, causaram porcentagens de fitotoxicidade que diferiram estatisticamente da testemunha. Entretanto, nota-se que ao longo do tempo, essas porcentagens de fitotoxicidade diminuíram de 66,25\% (7 DAE) para $37,50 \%$ (35 DAE) para o tratamento isoxaflutole e de 58,75\% (7 DAE) para 36,25\% (35 DAE) para o tratamento de isoxaflutole + indaziflam. Porém, estes tratamentos continuaram diferentes estatisticamente da testemunha, evidenciando que são tratamentos que causaram injúrias as plantas de cana-de-açúcar da variedade RB92579 provenientes de minirrebolos (Tabela 6).

Tabela 6. Fitotoxicidade (\%) de herbicidas pré-emergentes em minirrebolos de cana-de-açúcar da variedade RB92579.

Table 6. Phytotoxicity (\%) of pre-emergent herbicides in sugarcane mini-wheels of the RB92579 variety.

\begin{tabular}{|c|c|c|c|c|c|c|c|c|c|c|}
\hline \multirow{2}{*}{ Tratamentos } & \multicolumn{10}{|c|}{ Dias após emergência da cana-de-açúcar (DAE) } \\
\hline & \multicolumn{2}{|c|}{7} & \multicolumn{2}{|c|}{14} & \multicolumn{2}{|c|}{21} & \multicolumn{2}{|c|}{28} & \multicolumn{2}{|c|}{35} \\
\hline amicarbazone & 0,00 & $\mathrm{bA}$ & 0,00 & $\mathrm{bA}$ & 2,50 & $\mathrm{bA}$ & 3,75 & $\mathrm{bA}$ & 3,75 & $\mathrm{bA}$ \\
\hline metribuzin & 0,00 & $\mathrm{bA}$ & 0,00 & $\mathrm{bA}$ & 0,00 & bA & 0,00 & bA & 0,00 & bA \\
\hline indaziflam & 0,00 & bA & 2,50 & $\mathrm{bA}$ & 5,00 & bA & 6,25 & bA & 6,75 & bA \\
\hline isoxaflutole & 66,25 & aA & 63,75 & $\mathrm{aA}$ & 61,25 & $\mathrm{aA}$ & 38,75 & $\mathrm{aB}$ & 37,50 & $\mathrm{aB}$ \\
\hline amicarbazone + indaziflam & 0,00 & $\mathrm{bA}$ & 1,25 & $\mathrm{bA}$ & 6,25 & $\mathrm{bA}$ & 6,75 & $\mathrm{bA}$ & 7,50 & bA \\
\hline metribuzin + indaziflam & 0,00 & bA & 0,00 & $\mathrm{bA}$ & 3,75 & bA & 5,00 & $\mathrm{bA}$ & 6,25 & bA \\
\hline isoxaflutole + indaziflam & 58,75 & $\mathrm{aAB}$ & 63,75 & $\mathrm{aA}$ & 67,50 & $\mathrm{aA}$ & 45,00 & $\mathrm{aBC}$ & 36,25 & $\mathrm{aC}$ \\
\hline testemunha & 0,00 & $\mathrm{bA}$ & 0,00 & $\mathrm{bA}$ & 0,00 & $\mathrm{bA}$ & 0,00 & $\mathrm{bA}$ & 0,00 & $\mathrm{bA}$ \\
\hline $\mathrm{F}_{\text {tratamento }}=242,92^{* *}$ & & & avaliacão $=$ & $4,23 *$ & & & & $\mathrm{t} \times$ aval $=$ & & \\
\hline $\mathrm{DMS}_{\text {tratamento }}=14,63$ & & & & & & & $\mathrm{MS}_{\text {avaliac }}$ & $0=13$ & & \\
\hline
\end{tabular}

** Significativo ao nível de 5\% de probabilidade pelo teste F; * Significativo ao nível de 1\% de probabilidade pelo teste F; C.V. - Coeficiente de variação. . DMS - Diferença mínima significativa a 5\%. Médias seguidas por letras iguais, minúsculas na coluna e maiúsculas na linha, não diferem entre si pelo teste de Tukey a 5\% de significância.

$\mathrm{Na}$ Tabela 7 estão apresentados os resultados de fitotoxicidade obtidos para a variedade RB966928 plantada no sistema de minirrebolos.

Observa-se que aos 7 DAE os tratamentos com aplicação de amicarbazone, isoxaflutole isolado e em associação com indaziflam causaram 20,00; 35,00 e 55,00\% de fitotoxicidade, respectivamente, sendo que apenas os tratamentos de isoxaflutole isolado e em associação com indaziflam que diferiram estatisticamente da testemunha. No decorrer das avaliações, estes três tratamentos tiveram redução nos sintomas fitotóxicos, e aos 35 DAE nenhum deles foi diferente da testemunha. O tratamento contendo aplicação de indaziflam isolado, apesar de não causar injúrias as plantas de cana-de-açúcar aos 7 DAE, proporcionou fitotoxicidade a partir dos 14 DAE que teve evolução ao decorrer das avaliações, sendo de $37,75 \%$ aos 35 DAE, diferindo estatisticamente de testemunha e evidenciando que este herbicida causou injúrias nas plantas estudadas. Os demais tratamentos não apresentaram sintomas fitotóxicos diferentes estatisticamente da testemunha sem aplicação (Tabela 7).

Tabela 7. Fitotoxicidade (\%) de herbicidas pré-emergentes em minirrebolos de cana-de-açúcar da variedade RB966928.

Table 7. Phytotoxicity (\%) of pre-emergent herbicides in sugarcane mini-wheels of the RB966928 variety.

\begin{tabular}{|c|c|c|c|c|c|c|c|c|c|c|}
\hline \multirow{2}{*}{ Tratamentos } & \multicolumn{10}{|c|}{ Dias após emergência da cana-de-açúcar (DAE) } \\
\hline & \multicolumn{2}{|c|}{7} & \multicolumn{2}{|c|}{14} & \multicolumn{2}{|c|}{21} & \multicolumn{2}{|c|}{28} & \multicolumn{2}{|c|}{35} \\
\hline Amicarbazone & 20,00 & $\mathrm{abA}$ & 5,00 & $\mathrm{bA}$ & 2,50 & $\mathrm{bA}$ & 6,25 & $\mathrm{bA}$ & 6,25 & $\mathrm{bA}$ \\
\hline Metribuzin & 0,00 & bA & 2,50 & $\mathrm{bA}$ & 3,12 & $\mathrm{bA}$ & 3,75 & $\mathrm{bA}$ & 5,00 & $\mathrm{bA}$ \\
\hline Indaziflam & 0,00 & $\mathrm{bB}$ & 7,50 & $\mathrm{bB}$ & 15,00 & $\mathrm{bAB}$ & 30,00 & $\mathrm{abA}$ & 37,75 & aA \\
\hline Isoxaflutole & 35,00 & $\mathrm{aA}$ & 30,00 & $\mathrm{abA}$ & 27,50 & abA & 27,50 & $\mathrm{abA}$ & 20,00 & abA \\
\hline amicarbazone + indaziflam & 0,00 & $\mathrm{bA}$ & 0,00 & bA & 5,00 & bA & 10,00 & $\mathrm{abA}$ & 11,25 & $\mathrm{abA}$ \\
\hline metribuzin + indaziflam & 0,00 & $\mathrm{bA}$ & 1,25 & $\mathrm{bA}$ & 2,50 & bA & 5,00 & bA & 6,25 & bA \\
\hline isoxaflutole + indaziflam & 55,00 & aA & 53,75 & aA & 50,00 & aA & 36,25 & $\mathrm{aAB}$ & 23,75 & $\mathrm{abB}$ \\
\hline Testemunha & 0,00 & $\mathrm{bA}$ & 0,00 & $\mathrm{bA}$ & 0,00 & $\mathrm{bA}$ & 0,00 & bA & 0,00 & $\mathrm{bA}$ \\
\hline $\mathrm{F}_{\text {tratamento }}=20,13^{* *}$ & \multicolumn{4}{|c|}{$\mathrm{F}_{\text {avaliaç̃o }}=\mathrm{NS}$} & \multicolumn{6}{|c|}{$\mathrm{F}_{\text {trat } \mathrm{xaval}}=1,66^{*}$} \\
\hline $\mathrm{DMS}_{\text {tratamento }}=30,98$ & & & & & & & $\mathrm{MS}_{\text {avalii }}$ & ão $=3$ & & \\
\hline \multicolumn{11}{|c|}{ C.V. $(\%)=17,23$} \\
\hline
\end{tabular}

** Significativo ao nível de 5\% de probabilidade pelo teste F; * Significativo ao nível de 1\% de probabilidade pelo teste F; NS Não significativo pelo teste F; C.V. - Coeficiente de variação. . DMS - Diferença mínima significativa a 5\%. Médias seguidas por letras iguais, minúsculas na coluna e maiúsculas na linha, não diferem entre si pelo teste de Tukey a 5\% de significância.

Para as avaliações biométricas das plantas oriundas de minirrebolos da variedade RB92579, os dados obtidos estão expressos na Tabela 8.

Para altura, nota-se que nenhum dos tratamentos estudados foi diferente estatisticamente de testemunha. Para a avaliação de diâmetro dos colmos, a análise de variância não foi significativa, ou seja, não houve diferença estatística entre os tratamentos estudados. Com relação a análise do teor de clorofila, apenas os tratamentos com aplicação de isoxaflutole isolado e em associação com indaziflam, diferiram estatisticamente da testemunha sem aplicação, evidenciando redução no teor de clorofila nas plantas tratadas com estes herbicidas. Para área foliar, apenas o tratamento de isoxaflutole + indaziflam causou redução da área foliar significativa quando comparada a testemunha. Já para análise de massa seca da parte aérea, apenas o herbicida isoxaflutole 
aplicado de forma isolado foi diferente da testemunha, enquanto que para análise de massa seca do sistema radicular, apenas os tratamentos contendo aplicação de amicarbazone e metribuzin, não foram diferentes estatisticamente quando comparados a testemunha sem aplicação (Tabela 8).

Tabela 8. Avaliações biométricas aos 35 DAE, de minirrebolos de cana-de-açúcar da variedade RB92579, após aplicação de herbicidas em pré-emergência.

Table 8. Biometric evaluations at 35 DAE, of mini sugarcane wheels of the RB92579 variety, after herbicide application in pre-emergence.

\begin{tabular}{|c|c|c|c|c|c|c|c|c|c|c|c|}
\hline Tratamentos & \multicolumn{2}{|c|}{$\begin{array}{l}\text { Altura } \\
(\mathrm{cm})\end{array}$} & $\begin{array}{c}\text { Diâmetro } \\
(\mathrm{cm})\end{array}$ & \multicolumn{2}{|c|}{$\begin{array}{l}\text { Clorofila } \\
\left(\mathrm{mg} \mathrm{cm}^{-2}\right)\end{array}$} & \multicolumn{2}{|c|}{$\begin{array}{l}\mathrm{AF}^{1} \\
\left(\mathrm{~cm}^{2}\right)\end{array}$} & \multicolumn{2}{|c|}{$\begin{array}{c}\mathrm{MSPA}^{2} \\
(\mathrm{~g})\end{array}$} & \multicolumn{2}{|c|}{$\begin{array}{c}\text { MSSR }^{3} \\
(\mathrm{~g})\end{array}$} \\
\hline Amicarbazone & 60,50 & $\mathrm{a}$ & 6,25 & 38,37 & $\mathrm{a}$ & 150,70 & $\mathrm{ab}$ & 1,81 & $\mathrm{a}$ & 1,49 & A \\
\hline Metribuzin & 58,50 & $a b$ & 5,87 & 36,77 & a & 162,50 & A & 1,28 & $a b c$ & 1,04 & $\mathrm{Bc}$ \\
\hline Indaziflam & 48,75 & $a b c$ & 6,12 & 33,75 & a & 66,65 & $\mathrm{~cd}$ & 0,98 & $\mathrm{bc}$ & 0,31 & De \\
\hline Isoxaflutole & 37,50 & c & 7,00 & 24,82 & b & 55,45 & $\mathrm{~cd}$ & 0,62 & c & 0,59 & De \\
\hline amicarbazone +indaziflam & 42,50 & bc & 5,50 & 35,80 & $\mathrm{a}$ & 88,12 & bcd & 1,18 & abc & 0,62 & De \\
\hline metribuzin + indaziflam & 41,25 & c & 5,50 & 34,20 & a & 88,07 & $\mathrm{~cd}$ & 1,10 & abc & 0,66 & $\mathrm{Cd}$ \\
\hline isoxaflutole + indaziflam & 36,00 & c & 6,00 & 24,92 & b & 51,65 & $\mathrm{D}$ & 0,67 & bc & 0,25 & $\mathrm{E}$ \\
\hline testemunha & 50,25 & $\mathrm{abc}$ & 7,12 & 37,05 & $\mathrm{a}$ & 117,87 & $\mathrm{abc}$ & 1,38 & $\mathrm{ab}$ & 1,23 & $\mathrm{Ab}$ \\
\hline $\mathrm{F}$ & \multicolumn{2}{|c|}{$6,26^{*}$} & NS & \multicolumn{2}{|c|}{$8,20 * *$} & \multicolumn{2}{|c|}{$9,09 * *$} & \multicolumn{2}{|c|}{$5,80^{*}$} & \multicolumn{2}{|c|}{$10,78^{* *}$} \\
\hline DMS & \multirow{2}{*}{\multicolumn{2}{|c|}{$\begin{array}{l}17,22 \\
1567\end{array}$}} & 2,10 & \multicolumn{2}{|c|}{8,76} & \multicolumn{2}{|c|}{65,89} & \multicolumn{2}{|c|}{0,75} & \multicolumn{2}{|c|}{0,39} \\
\hline C.V. $(\%)$ & & & 14,53 & \multicolumn{2}{|c|}{11,26} & \multicolumn{2}{|c|}{29,00} & \multicolumn{2}{|c|}{28,30} & \multicolumn{2}{|c|}{24,55} \\
\hline
\end{tabular}

${ }^{1} \mathrm{AF}$ - Área foliar; ${ }^{2} \mathrm{MSPA}$ - Massa seca da parte aérea; ${ }^{3}$ MSSR - Massa seca do sistema radicular. ${ }^{* *}$ Significativo ao nível de $5 \%$ de probabilidade pelo teste F; * Significativo ao nível de 1\% de probabilidade pelo teste F; NS Não significativo pelo teste F; C.V. - Coeficiente de variação. DMS - Diferença mínima significativa a 5\%. Médias seguidas por letras iguais não diferem entre si pelo teste de Tukey a 5\% de significância.

Já os resultados obtidos para as avaliações biométricas das plantas oriundas de minirrebolos da variedade RB966928 estão expressos na Tabela 9.

Observa-se que para avaliação de altura, apenas o tratamento de indaziflam isolado resultou em redução significativa na altura das plantas quando comparado com a testemunha. Já para as avaliações do diâmetro dos colmos e do teor de clorofila nas folhas, apenas a associação de metribuzin + indaziflam proporcionou redução do diâmetro, e a associação de isoxaflutole + indaziflam causou redução no teor de clorofila, diferindo estatisticamente da testemunha. Para as análises de área foliar e massa seca da parte aérea, nenhum dos tratamentos herbicidas diferiu estatisticamente da testemunha sem aplicação. Já para a massa seca do sistema radicular, o tratamento com aplicação de indaziflam isolado reduziu o peso do sistema radicular, sendo diferentes da testemunha estatisticamente (Tabela 9).

Tabela 9. Avaliações biométricas aos 35 DAE, de minirrebolos de cana-de-açúcar da variedade RB966928, após aplicação de herbicidas em pré-emergência.

Table 9. Biometric evaluations at 35 DAE, of mini sugarcane wheels of the RB966928 variety, after application of pre-emergence herbicides.

\begin{tabular}{|c|c|c|c|c|c|c|c|c|c|c|c|c|}
\hline Tratamentos & \multicolumn{2}{|c|}{$\begin{array}{c}\text { Altura } \\
(\mathrm{cm})\end{array}$} & \multicolumn{2}{|c|}{$\begin{array}{l}\text { Diâmetro } \\
(\mathrm{cm})\end{array}$} & \multicolumn{2}{|c|}{$\begin{array}{l}\text { Clorofila } \\
\left(\mathrm{mg} \mathrm{cm}^{-2}\right)\end{array}$} & \multicolumn{2}{|c|}{$\begin{array}{l}\mathrm{AF}^{1} \\
\left(\mathrm{~cm}^{2}\right)\end{array}$} & \multicolumn{2}{|c|}{$\begin{array}{c}\text { MSPA }^{2} \\
(\mathrm{~g})\end{array}$} & \multicolumn{2}{|c|}{$\begin{array}{c}\text { MSSR }^{3} \\
(\mathrm{~g})\end{array}$} \\
\hline Amicarbazone & 50,75 & $\mathrm{ab}$ & 6,62 & $\mathrm{ab}$ & 44,97 & $\mathrm{a}$ & 108,85 & $\mathrm{ab}$ & 1,24 & $\mathrm{ab}$ & 1,74 & A \\
\hline Metribuzin & 51,00 & $\mathrm{ab}$ & 5,50 & $a b$ & 43,75 & a & 100,80 & $a b$ & 1,16 & $a b$ & 1,66 & A \\
\hline Indaziflam & 33,00 & $\mathrm{~b}$ & 7,37 & $\mathrm{a}$ & 36,65 & $a b$ & 65,00 & $a b$ & 1,06 & $a b$ & 0,24 & $\mathrm{C}$ \\
\hline Isoxaflutole & 49,25 & $\mathrm{ab}$ & 7,25 & a & 42,85 & $\mathrm{a}$ & 134,46 & $\mathrm{a}$ & 1,64 & a & 1,72 & A \\
\hline amicarbazone +indaziflam & 55,25 & a & 6,50 & $\mathrm{ab}$ & 42,67 & a & 121,00 & $\mathrm{ab}$ & 1,64 & a & 0,86 & $a b c$ \\
\hline metribuzin + indaziflam & 48,50 & $\mathrm{ab}$ & 4,75 & $\mathrm{~b}$ & 42,00 & a & 97,47 & $\mathrm{ab}$ & 1,49 & a & 0,43 & bc \\
\hline isoxaflutole + indaziflam & 39,25 & $\mathrm{ab}$ & 6,62 & $a b$ & 30,20 & $\mathrm{~b}$ & 42,25 & $\mathrm{~b}$ & 0,55 & $\mathrm{~b}$ & 0,42 & bc \\
\hline Testemunha & 54,50 & $\mathrm{a}$ & 7,12 & a & 42,27 & a & 94,67 & $\mathrm{ab}$ & 1,31 & $\mathrm{ab}$ & 1,41 & $\mathrm{ab}$ \\
\hline $\mathrm{F}$ & \multicolumn{2}{|c|}{$1,17^{*}$} & \multicolumn{2}{|c|}{$1,80^{* *}$} & \multicolumn{2}{|c|}{$4,24 * *$} & \multicolumn{2}{|c|}{$2,21 *$} & \multicolumn{2}{|c|}{$1,47 * *$} & \multicolumn{2}{|c|}{$7,76^{* *}$} \\
\hline DMS & \multicolumn{2}{|c|}{33,35} & \multicolumn{2}{|c|}{1,98} & \multicolumn{2}{|c|}{11,10} & \multicolumn{2}{|c|}{84,01} & \multicolumn{2}{|c|}{0,86} & \multicolumn{2}{|c|}{1,08} \\
\hline C.V. (\%) & \multicolumn{2}{|c|}{29,86} & \multicolumn{2}{|c|}{20,99} & \multicolumn{2}{|c|}{11,65} & \multicolumn{2}{|c|}{21,73} & \multicolumn{2}{|c|}{26,94} & \multicolumn{2}{|c|}{23,47} \\
\hline
\end{tabular}

${ }_{1}^{1} \mathrm{AF}$ - Área foliar; ${ }^{2} \mathrm{MSPA}$ - Massa seca da parte aérea; ${ }^{3}$ MSSR - Massa seca do sistema radicular. $* *$ Significativo ao nível de $5 \%$ de probabilidade pelo teste F; * Significativo ao nível de $1 \%$ de probabilidade pelo teste F; C.V. - Coeficiente de variação. DMS - Diferença mínima significativa a 5\%. Médias seguidas por letras iguais não diferem entre si pelo teste de Tukey a $5 \%$ de significância.

\section{DISCUSSÃO}

Os resultados obtidos indicam que a associação dos herbicidas amicarbazone, metribuzin e isoxaflutole com o herbicida indaziflam, podem resultar em efeito sinérgico nas plantas de cana-de-açúcar das variedades estudadas, ocasionando danos fitotóxicos. Assim como o indaziflam utilizado de forma isolada, que pode resultar em severa fitotoxicidade. Esse comportamento pode ocorrer em função de dois fatores, o primeiro relativo as características físicoquímicas do indaziflam e o segundo, relativo ao posicionamento do sistema radicular no sistema de plantio.
Em relação às características físico-químicas do indaziflam, segundo Alonso et al. (2011), esse herbicida apresenta baixa mobilidade no solo em detrimento dos seus elevados valores de coeficiente de adsorção $(\mathrm{Kd})$, baixo coeficiente de adsorção normatizado para o teor de matéria orgânica (Koc) e alto valor do coeficiente de partição ocatnol-água ( $\log$ de Kow $=2,88)$, que classifica esse herbicida como muito lipofílico. Essas características do indaziflam resultam em baixa mobilidade vertical e lixiviação desse herbicida no solo. 
Associado a isso, temos que no sistema de plantio através de mudas pré-brotadas de cana-de-açúcar (MPB) e minirebolos, o sistema radicular é posicionado diretamente na zona tratada com os herbicidas, camadas de profundidade inicial do perfil do solo (primeiros $10,0-20,0 \mathrm{~cm}$ ), logo, o sistema radicular fica alocado na área com maior concentração do herbicida. Portanto, o indaziflam, que apresenta baixa mobilidade, pode ter ficado em maior concentração próximo ao sistema radicular das MPBs e minirebolos, resultando em maior absorção e translocação do indaziflam e, consequentemente, efeitos fitotóxicos nas plantas de cana-de-açúcar. Sendo a sintomatologia inerente a exposição ao indaziflam crescente, indicando constante absorção desse produto, uma vez que ele provavelmente não foi degradado e/ou transportado da zona radicular.

Resultados semelhantes foram encontrados por Hijano (2016) ao estudar a seletividade do indaziflam e a associação de indaziflam + metribuzim em MPB que constatou para as aplicações realizadas em pré-plantio de indaziflam isolado (37,5 a 112,5 $\left.\mathrm{g} \mathrm{ha}^{-1}\right)$ e na associação de indaziflam (37,5 a 112,5 $\mathrm{g} \mathrm{ha}^{-1}$ ) com metribuzim (480 a $1440 \mathrm{~g} \mathrm{ha}^{-1}$ ), morte das mudas das variedades RB966928 e CTC14.

Independentemente da variedade estudada, o isoxaflutole isolado e/ou associado com indaziflam, resultou em expressivos efeitos fitotóxicos em cana-de-açúcar, os quais se tornaram mais severos ao longo dos períodos de avaliação para MPBs e menos severos para minirebolos. O isoxaflutole se trata de um pró-herbicida, ou seja, a molécula de herbicida biologicamente ativa no controle de plantas daninhas se trata da dikitonitrila $(\mathrm{DKN})$, essa conversão ocorre rapidamente em ambientes com alta umidade. Por essa conversão o isoxaflutole normalmente é posicionado em períodos de seca na cultura da cana-de-açúcar (RODRIGUES; ALMEIDA, 2018). Nesse experimento a irrigação diária foi de $10 \mathrm{~mm}$, favorecendo a disponibilidade dessa molécula em solução do solo para absorção do sistema radicular da cana-de-açúcar, fato que justifica a maior porcentagem de fitotoxicidade inicial.

Azania et al. (2006), trabalhando com a aplicação de isoxaflutole e metribuzin em cana-planta, observaram que esses produtos resultaram em efeitos mais fitotóxicos à canade-açúcar até os 15 DAA (Dias Após a Aplicação), porém aos 30 e 45 DAA apresentaram-se como os menos tóxicos em relação aos tratamentos estudados, corroborando com os dados obtidos no presente experimento para minirrebolos.

Bertolino; Alves (2014) ao avaliar a fitotoxicidade de herbicidas no sistema de minirrebolos (Plene $($ ) $)$ para a variedade SP81-3250, em pré-emergência, constataram que o isoxaflutole $\left(350 \mathrm{~g} \mathrm{ha}^{-1}\right)$ não foi seletivo. Os autores atribuem os maiores efeitos de toxidez em pré-emergência ao fato de os toletes possuírem locais de absorção expostos (nas partes cortadas do tolete, próximas às gemas), fato que poderia ter comprometido o desenvolvimento das gemas e, por consequência, da planta emersa.

Os herbicidas amicarbazone e metribuzin, representam uma alternativa mais segura em relação a seletividade em cana-planta em MPBs e minirrebolos para o controle de plantas daninhas, por ocasionarem redução de fitotoxicidade ao longo do tempo e/ou efeitos iniciais relativamente baixos. $\mathrm{O}$ amicarbazone e metribuzin apresentam alta solubilidade em água (4600 mg L-1 (pH 4-9) e $1100 \mathrm{mg} \mathrm{L}^{-1}$ (a 20 C), respectivamente) e baixo Kow (RODRIGUES; ALMEIDA, 2018). Tais características favorecem sua maior lixiviação para camadas mais além da faixa de concentração do sistema radicular, diminuindo a absorção desses produtos e reduzindo os efeitos fitotóxicos.

\section{CONCLUSÕES}

Para as variedades de mudas pré-brotadas (MPBs) RB966928 e IAC 5000, somente os herbicidas amicarbazone e metribuzin foram seletivos. Já para os minirebolos de canade-açúcar, os tratamentos com isoxaflutole isolado e em associação com indaziflam não foram seletivos para a variedade RB92579, e para a variedade RB966928 os tratamentos com isoxaflutole e indaziflam, isolados e em associação, não foram seletivos. O herbicida metribuzim foi seletivo em nos dois experimentos para todas as variedades.

\section{AGRADECIMENTOS}

A FAPESP (Fundação de Amparo à Pesquisa do Estado de São Paulo), pelo financiamento dessa pesquisa através da bolsa de iniciação cientifica como consta no processo $n^{\circ}$ 2015/14833-0.

\section{REFERÊNCIAS}

ALONSO, D. G.; KOSKINEN, W. C.; OLIVEIRA, R. S.; CONSTANTIN, J.; MISLANKAR, S. Sorptiondesorption of indaziflam in selected agricultural soils. Journal of Agricultural and Food Chemistry, Easton, v. 59, n. 24, p. 13096-13101, 2011. DOI: https://dx.doi.org/10.1021/jf203014g

ARAUJO, R. B. Avaliação de diferentes tipos de propágulos no desenvolvimento inicial da cana-deaçúcar (Saccharum officinarum L.). 2015. 102f. Dissertação (Mestrado em Ciências) - Universidade de São Paulo, Piracicaba, 2015.

AZANIA, C. A. M.; ROLIM, J. C.; CASAGRANDE, A. A.; LAVORENTI, N. A. I.; AZANIA, A. A. P. M. Seletividade de herbicidas: III - aplicação de herbicidas em pós emergência inicial e tardia da cana-de-açúcar na época da estiagem. Planta daninha, Viçosa, v. 24, n. 3, p. 489-495, 2006. DOI: https://dx.doi.org/10.1590/S0100-83582006000300010

BARBOSA, J. C.; MALDONADO JR, W. Experimentação Agronômica e AgroEstat - Sistema para Análises Estatísticas de Ensaios Agronômicos. Jaboticabal: Gráfica Multipress Ltda, 2015. 396 p.

BERTOlinO, C. B.; ALVES, P. L. C. A. Seletividade de herbicidas para cana-de-açúcar no sistema Plene $\AA^{\circledR}$ em pré e pós-emergência. Revista Brasileira de Herbicidas, Londrina, v. 13, n. 2, p. 197-206, 2014. DOI: https://dx.doi.org/10.7824/rbh.v13i3.249

DIAS, J. L. C. S.; SILVA JUNIOR, A. C.; QUEIROZ, J. R. G.; MARTINS, D. Herbicides selectivity in pre-budded seedlings of sugarcane. Arquivos do Instituto Biológico, São Paulo, v. 84, e0112015, 2017. DOI: https://dx.doi.org/10.1590/1808-1657000112015

EMBRAPA_EMPRESA BRASILEIRA DE PESQUISA AGROPECUÁRIA. Sistema brasileiro de classificação de solos. Brasília: Embrapa Produção de Informação/Embrapa Solos, 2013. 154 p.

EWRC_EUROPEAN WEED RESEARCH COUNCIL. Report of the 3rd and 4th meetings of EWRC Comittee of Methods in Weed Research. Weed Research, v. 4, p. 88, 1964. 
FRAGA JUNIOR, E. F. Considerações sobre o manejo de irrigação na produtividade e qualidade de gemas de cana-de-açúcar para viveiros de mudas-prébrotadas (MPB). 2015. 111f. Tese (Doutorado em Engenharia de sistemas Agrícolas) - Escola Superior de Agricultura Luiz de Queiroz, Universidade de São Paulo, Piracicaba. 2015.

GIRALDELI, A.; SILVA, A.; BRITO, F.; ARAÚJO, L.; PAGENOTTO, A.; MORAES, J.; VICTORIA FILHO, R. Crescimento inicial de mudas pré-brotadas de cana-deaçúcar em duas modalidades de aplicação de herbicidas. Revista Brasileira de Herbicidas, Londrina, v. 17, n. 3, e588, 2018.

DOI: https://dx.doi.org/10.7824/rbh.v17i3.588

HIJANO, N. Interferência de capim-camalote em canade-açúcar e seletividade de indaziflam e indaziflam + metribuzin aplicados em cana-de-açúcar no sistema MPB. 2016. 112f. Dissertação (Mestrado em Produção Vegetal) - Universidade Estadual Paulista, Faculdade de Ciências Agrárias e Veterinárias, Jaboticabal, 2016.

LANDELL, M. G. A.; CAMPANHA, M. P.; FIGUEIREDO, P.; XAVIER, M. A.; ANJOS, I. A.; DINARDO-MIRANDA, L. L. Sistema de
Multiplicação de cana-de-açúcar com uso de mudas pré-brotadas (MPB) oriundas de gemas individualizadas. Campinas: Instituto Agronômico, 2013. 109 v. 16 p. (Documentos IAC, 109).

MARTINHO, L.; BOCCHI, M.; JEPSON, I.; MOREIRA, M.; CARVALHO, J. C. Plene, an innovative approach for sugarcane planting in Brazil. Proceedings International Society of Sugar Cane Technologists, v. 27, p. 1-10, 2010.

MAWLA, H. A.; HEMIDA, B.; MAHMOUD, W. A. Study on the mechanization of sugar cane transplanting. International Journal of Engineering and Technical Research, v. 2, n.8, 2014.

MAY, A.; RAMOS, N. P. Uso de gemas individualizadas de cana-de-açúcar para a produção de mudas. Jaguariúna: Embrapa, 2019. 29 p. (Circular técnica, 29)

RAIJ, B. V.; CANTARELLA, H.; QUAGGIO, J. A.; FURLANI, A. M. C. Recomendações de adubação e calagem para o Estado de São Paulo. 2. ed. Campinas: Instituto Agronômico; Fundação IAC, 1996. 285 p. (Boletim Técnico, 100).

RODRIGUES, B. N.; ALMEIDA, F. S. Guia de herbicidas. 7. ed. Londrina: Edição dos autores, 2018. 764 p. 